\title{
Spezifische Immuntherapie
}

\section{Neue Daten, neue Optionen}

\author{
Bei allergischen Erkrankungen ist die spezifische Immuntherapie \\ essenzieller Bestandteil des Behandlungsregimes. Eine Vielzahl aktueller \\ Publikationen bestätigt mittlerweile die Wirksamkeit der sublingualen \\ Applikation. Einen Überblick verschaffte der Vortrag von Prof. Dr. Walter \\ Canonica auf der Veranstaltung „Allergologie im Kloster".
}

\begin{abstract}
$\mathrm{n}$ vielen Fachbereichen der Medizin konzentriert sich die Forschung zunehmend auf bestimmte Phänotypen einer Erkrankung. Im Zentrum stehen dabei oft patientenspezifische Strukturen oder Substanzen, die auf molekularer Ebene pathogen wirken. Prof. Dr. Walter Canonica, Universität Genua, ehemaliger Präsident der World Allergy Organization (WAO), wies darauf hin, dass dieses Phänotyp-orientierte Konzept auf allergische Erkrankungen genau zutrifft: „Der immunologische Mechanismus ist bekannt, das spezifische auslösende Agens ist bekannt und wir haben die Biomarker, um es zu de-
\end{abstract}

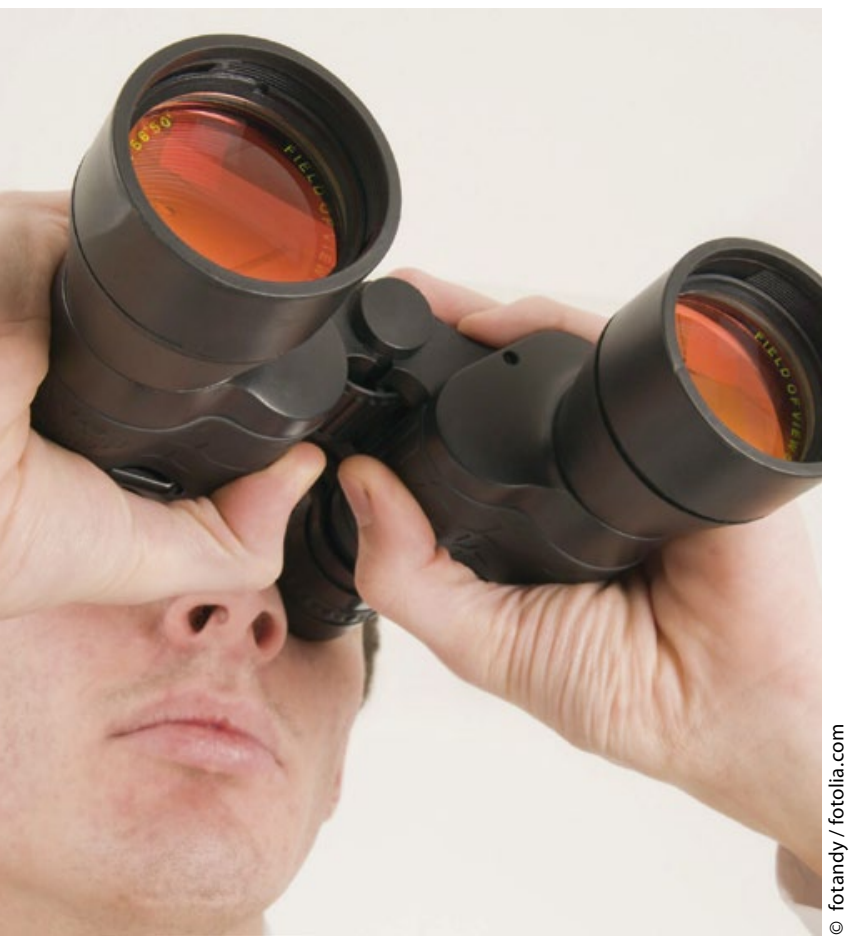

Blick in die Zukunft der Immuntherapie: SCIT und SLIT sind gemeinsam besonders effektiv. finieren“. Die allergenspezifische Sensibilisierung bei allergischem Asthma oder allergischer Rhinokonjunktivitis stelle einen Phänotyp, sogar einen „Prototyp des Phänotyps“ dar.

\section{Effektive SLIT}

Canonica machte auf ein Review der Cochrane Collaboration aufmerksam, in der die Autoren die sublinguale Immuntherapie (SLIT) als effektive und sichere Behandlung der allergischen Rhinitis bestätigen [Radulovic $S$ et al. Cochrane Database Syst Rev 2010, Dec 8]. Er gab zudem eine Übersicht über aktuelle Publikationen aus aller Welt, die die Wirksamkeit der SLIT belegen. Als aktuellste Studie nannte er die Arbeit von Creticos et al., die diese Therapie als erfolgreiche und verträgliche Behandlung bei Allergie gegen Ambrosia (Ragweed) vorstellt. Das Sicherheitsprofil erlaubte bei den Patienten eine tägliche Applikation des Major-Allergens Amb a 1-U [Creticos PS et al. J Allergy Clin Immunol 2013; 131: 1342-9].

\section{SLIT am besten für vier Jahre?} In einer prospektiven Vergleichsstudie über 15 Jahre prüften Marogna et al. die Effekte einer drei-, vier- oder fünfjährigen SLIT bei Patienten mit Hausstaubmilbenallergie [Marogna $\mathrm{M}$ et al. J Allergy Clin Immunol 2010; 126: 96975]. Der Verbrauch an antiallergischer Medikation ging in allen Studienarmen zunächst gegenüber der Kontrollgruppe deutlich zurück. Waren die Patienten drei Jahre lang hyposensibilisiert worden, zeigte sich nach etwa sieben
Jahren ein Wiederanstieg von Bedarfsmedikation und klinischen Scores. Nach vier- und fünfähriger Hyposensibilisierung war dieser Anstieg geringer und um etwa ein Jahr verzögert. Eine erneute SLIT führte zu einem raschen Abklingen der Symptome, der Effekt trat hier schneller ein als bei der ersten Immuntherapie.

Canonica verwies zudem auf ein aktuelles systematisches Review von Dretzke et al., die die sublinguale der subkutanen Immuntherapie (SCIT) bei saisonaler allergischer Rhinitis gegenüberstellten [Dretzke J et al. J Clin Allergy Clin Immunol 2013; 131: 1361-6]. Die Autoren kamen zu dem Schluss, dass sich für keine der beiden Applikationsformen ein konsistenter Vorteil im Hinblick auf Symptome, Bedarfsmedikation oder Lebensqualität ergab, wenngleich ein Trend zugunsten der subkutanen Form bei den Symptom-Medikations-Scores erkennbar war.

\section{Kombination möglich?}

Nach den Ergebnissen einer weiteren Studie lassen sich SCIT und SLIT vermutlich auch gut kombinieren. Kollegen in Istanbul verglichen bei Kindern mit Hausstaubmilben-induziertem Asthma die folgenden Gruppen: Pharmakotherapie, SCIT, SLIT und die Kombination beider Immuntherapien [Keles $\mathrm{S}$ et al. J Allergy Clin Immunol 2011; 128: 808-15. e7]. Beide Immuntherapien, so Canonica, waren effektiver als die pharmakologische Therapie. Die Kombination SCIT plus SLIT zeigte eine größere Effektivität als die SLIT und ein besseres Sicherheitspotenzial als die SCIT. Die Autoren sehen in den Ergebnissen ihrer Arbeit die Möglichkeit zur schnelleren und effektiveren Auftitrierung durch die subkutane Therapie und zur sichereren Erhaltungsphase mit der sublingualen Behandlung.

Dr. Andreas Fischer

Canonica GW "How is the world performing allergen-specific immunotherapy? The WAO perspective" im Rahmen des interdisziplinären Symposiums "Allergologie im Kloster", EltvilleEberbach, 25. Mai 2013 